\title{
Introduction to Pancreatic Cancer
}

\author{
Georgios P. Fragulidis
}

\subsection{Introduction}

The goal of pancreatic imaging is the early detection and characterization of clinically relevant pancreatic lesions. Incidental pancreatic lesions are increasingly common and can range from benign incidental lesions to malignant. Consequently, in some cases the detection of a mass on imaging is nonspecific, and $5-15 \%$ of pancreatic resections may show benign pathology given the significant overlap of patient symptoms in benign and malignant pancreatic disorders. In addition, no pancreatic mass is visualized on imaging evaluation in $10 \%$ of cases since the tumor may be isoattenuating [1]. Thus, the presence and location of a mass may be assumed from secondary signs such as mass effect, an abnormal convex contour of the pancreas, ductal obstruction, and vascular invasion. Therefore, the use of a multimodality approach combines the strengths of individual imaging modalities and has a synergistic effect in improving diagnostic yield.
Imaging techniques currently used for diagnosis and preoperative staging of PC include transabdominal ultrasound (US), contrast-enhanced multidetector computed tomography (MDCT), magnetic resonance imaging (MRI), MR cholangiopancreatography (MRCP), and PET scan and invasive imaging modalities like endoscopic ultrasound (EUS), endoscopic retrograde cholangiopancreatography (ERCP), and intraductal ultrasound (IDUS) [2].

\section{References}

1. Sharma C, Eltawil KM, Renfrew PD, Walsh MJ, Molinari M (2011) Advances in diagnosis, treatment and palliation of pancreatic carcinoma: 1990-2010. World J Gastroenterol 17(7):867-897. https://doi. org/10.3748/wjg.v17.i7.867

2. Coté GA, Smith J, Sherman S, Kelly K (2013) Technologies for imaging the normal and diseased pancreas. Gastroenterology 144(6):1262-71.e1. https://doi.org/10.1053/j.gastro.2013.01.076

\footnotetext{
G. P. Fragulidis, $\mathrm{MD}, \mathrm{PhD}$

2nd Department of Surgery, National and

Kapodistrian University of Athens School of

Medicine, 76, Vas. Sofias str., Athens 11528

Attica, Greece

e-mail: gfragulidis@ aretaieio.uoa.gr
} 